\title{
On region based inference in genome wide association study
}

\author{
Sergey V. Malov \\ Theodosius Dobzhansky Center for \\ Genome Bioinformatics \\ St.-Petersburg State University \\ St-Petersburg, Russia \\ ORCID: 0000-0003-0093-6506
}

\author{
Alexey Antonik \\ Theodosius Dobzhansky Center for \\ Genome Bioinformatics \\ St.-Petersburg State University \\ St-Petersburg, Russia \\ ORCID: 0000-0003-4341-1291
}

\author{
Andrey K. Shevchenko \\ Theodosius Dobzhansky Center for \\ Genome Bioinformatics \\ St.-Petersburg State University \\ St-Petersburg, Russia \\ ORCID: 0000-0002-7332-5661
}

\begin{abstract}
We consider an advanced framework for genome wide association study (GWAS) based on the signal localization. Instead of looking for single genetic markers associated with the phenotype we attempt to discover regions of genetic markers, which are associated with phenotype. We focus on the localization by moving sums of negative logarithms of p-values, which is efficient for discovery of wide regions of genetic markers associated with phenotype. Particularly, we expect the method should be efficient for searching genes containing a number of genetic markers, if any reconstruction in a gene related to a phenotype. The method is implemented in GWATCH software for visualization and interpretation results of multiple statistical tests for genome associations. We discuss some features of the GWATCH software and apply them for HIV/AIDS cohort study from Botswana.
\end{abstract}

\section{Keywords - GWAS, signal localization, chi-square test}

\section{Introduction}

Genome wide association study (GWAS) is one of the most common and important methods is genetic research, set up on the statistical conclusions based on the results of multiple statistical tests. The aim aim of the GWAS is to discover genetic markers associated with the observed feature (phenotype). Increasing the number of awailable genetic markers leads to increasing statistical corection that makes it impossible reliable discovery of the associations. We consider an advanced method in GWAS based on signal localization [1]. Instead of looking for the single genetic markers associated with the phenotype we attempt to discover regions of genetic markers, which are associated with phenotype. The method is not efficient for associations related to single markers, but it tends to be efficient when a sufficient number of markers associated with phenotype within the same region of genome. For example our method is expected to be efficient if any reconstruction in a gene containing a number of genetic markers leads to phenotype changing. Genome Wide Tracks Chromosome Highway (GWATCH) is the advanced statistical software designed to automate the GWAS data analysis, visualization of results and meta-analysis. We discuss features of GWATCH related to region-based association discovery with some applications for real data analysis.

\section{Signal localization method}

Signal localization is quite a general methodology combining signal detection and signal indentification methods.

\section{The signal localization framework}

Let $H_{i}, i=1, \ldots, \mathrm{n}$ be the set of null hypotheses. Introduce a family of localization sets $\mathrm{W}=\left\{W_{j}\right\}_{j=1}^{k}$, where each of the localization sets $W_{j}$ are some subsets of indices, $\mathrm{j}=1, \ldots, \mathrm{k}$.
The localization hypothesis $\widetilde{H_{J}}$ holds, if all of the hypotheses $H_{i}, i \in W_{j}$ holds. Rejection of $\widetilde{H_{j}}$ means that there is at least one association (a signal) in the localization set $W_{j}$. Note that if $H_{i}$ is the hypothesis of independence between the observed feature $\mathrm{Y}$ and the control variable $X, i=1, \ldots, n$, the localization hypothesis is weaker than the hypothesis of full independence between $Y$ and the vector $\left(X_{i}, i \in W_{j}\right)$, and, therefore, methods which are capable of testing the hypothesis of full independence are applicable to test the localization hypothesis as well, but may be more conservative. Choice of the localization family is rather variative. The methods of controlling an error in multiple testing remains important in the localization problem. Enriching the family of localization sets tightens the multiple testing correction, whereas selection of poor localization family is quite subjective in most cases, and user should posses a valuable arument for that choice (e.g. disjoint regions of successive markers of size $k$ looks speculative, but selection genes as the localization setes may be natural in some cases). Further we use moving window localization, which is quite objective, and multiple testing correction does not exceed one from the classical GWAS. Let $T_{i}$ be a test statistic capable of testing the null hypothesis $H_{i}, p_{i}$ be the corresponding $\mathrm{p}$-value, $i=1, \ldots, \mathrm{n}$. It looks natural to apply methods of meta-analysis based on the p-values $p_{i}, i \in W_{j}$ for testing the localization hypothesis $\widetilde{H}_{J}, j=1, \ldots, k$. Fisher and Tippett approaches are applicable for testing the localization problem.

\section{Localization using Fisher's statistic}

Usage of the Fisher's combined probability method for the moving localization sets with the same number of available markers leads to the moving average process. Another choice is to use moving localization windows of the same length. In both cases the multiple testing correction is comparable with one from the classical GWAS, and it cannot be sufficiently improved in general case. The distribution of the Fisher's combined test statistic for independent markers is well known, but if the genetic markers are in linkage disequilibrium the true distribution of the statistic is not available. The naive $p$-values obtained under independence assumption are less then the true (adjusted) ones in most cases of dependence. Moreover, in most of practical cases the naive p-values are not srtictly related to the adjusted ones. Using the naive instead of adjusted $\mathrm{p}$-values may lead to false discoveries.

The distribution of the Fisher's statistic under full independence hypotesis can be evaluated by using random permutation method. The random permutation method over all localization windows requires a lot of computations and we are using the adaptive approach as discussed in [1], but even doing that it is unattainable to obtain all the adjusted pvalues. In order to discover the most significant regions we 
are using the three step algorithm: (i) obtaining the naive pvalues; (ii) selecting top hits of the localization windows with smallest naive p-values (e.g. top 5000), or with the naive $\mathrm{p}$-values not exceeding some fixed threshold (e.g. $10^{-8}$ ), and calculating adjusted $\mathrm{p}$-values for the selected localization windows; (iii) calibrating the smallest adjusted $\mathrm{p}$-values in order to eliminate their bias related to the random permutation method.

\section{Localization using $\chi^{2}$}

In a special case of binary phenotype and binary genetic marker (e.g. the domonant, the recessive model or the allelic approach) we developed the signal localization method based on $\chi^{2}$ distribution. The chi-square test statistic for the $2 \times 2$ contingency table is actually a square of asymptotically normal statistic $\xi$. For each genetic marker in a fixed localization window we calculate the asymptotically normal statistic $\xi_{i}$ and estimate the asymptotic correletion matrix $\Sigma$ of the random vector $\xi=\left(\xi_{1}, \ldots, \xi_{s}\right)$, where $s$ is a size of the localization window. Now, using the convergence $\xi^{T} \Sigma^{-1} \xi \Rightarrow \chi_{s}^{2}$ we obtain the adjusted p-value. Poor estimation quality of the correlation matrix for wide localization windows is an open problem of this method. For reasonable sizes of localization windows the method is much faster then the perutation one, and it allows to compute adjusted $p$-values for all the localization windows.

\section{GWATCH statistical software}

Genome Wide Association Tracks Chromosome Highway (GWATCH) is the interactive software designed for efficient computation of the multiple tests on association between phenotype and any genotype available, advanced $3 \mathrm{D}$ visualization and automated analysis of multiple testing results as well as its meta-analysis [2]. The GWATCH as its input accepts both original genotypes and clinical data to calculate multiple statistical tests for further analysis, and pre-computed results of multiple tests supplied by the user. In the current version only the categorical tests (combined exact Fisher's test and $\chi^{2}$ ) and the linear regression model are implemented for multiple tests computation. Other types of tests should be pre-computed by user and imported into GWATCH. We are planning to extend the list of multiple tests (first of all, for GLM and survival data analysis) available for computation in the future.

\section{Main features}

The 3-D highway vizualization concept is implemented on the top of WebGL API and renders navigable 3D view of the SNP data, which assists user to identify regions of high statistical significance and, therefore, to warrant any further investigation. Results of different statistical tests on the associations for large number of SNP's are produced and visualized in the form of colored 3D bars located according to the SNP position in genome and the type of statistical test. The height of a bar corresponds to the P-value for that SNP and test, and the color indicates the association nature (positive or negative), which determined by the sign of quantative association statistic (QAS). Genes related to SNP's are displayed along the highway. Then user can interactively retrieve the important information on the selected genetic marker (SNP), generate TRAX report which contains explanatory analysis of the genotype-phenotype association and complete list of results of the statistical tests available for the selected marker, as well as various reports based on tests results in the neghbourhood of the selected SNP in either SVG or PDF format.

\section{Top hits and the localization}

The baseline top hits tables contains the most significant p-values, top values of the QAS, top mean negative log pvalues and top of the naive P-values for several types of localization windows. These tables are assembled automatically after data loading and test calculation. User can enrich the two last types of top hit tables by adding the adjusted $\mathrm{p}$-values.

\section{Applications}

Botswana has the third highest HIV prevalence in the world (UNAIDS, 2019). We use GWATCH software to identify single variants and regions of genome demonstrating noticable association with HIV-1 subtype $\mathrm{C}$ infection in a Botswana cohort using a combination of whole-genome sequencing (WGS) and microarray genotype data. The genetic and clinical data for two cohorts were obtained in collaboration with Harvard T. H. Chan School of Public Health AIDS Initiative (Boston, USA) and Botswana Harvard AIDS Institute (Gaborone, Botswana). As a result, we have replicated several loci that have shown association with HIV in previous studies [3]. In addition, we have identified several novel putative associated loci slightly below the statistical significance threshold. Applying the localization by moving average with the window sizes of 21 , $41,71,201$ loci, $100 \mathrm{Kbp}$ and $200 \mathrm{Kbp}$ we obtain using the WGS dataset two novel putative regions associated significantly with the HIV-1C infection. The multiple testing correction is evaluated by simulations under the assumption of independent tests.

\section{ACKNOWLEDGMENT}

This research was supported in part by St.-Petersburg State University (Grant № 51148284) and by RSF (Grant Agreement № 20-14-00072).

\section{REFERENCES}

[208] S.V. Malov, A. Antonik, M. Tang, A. Berred, Yi Zeng and S.J. OBrien "Signal localization: a new approach in signal discovery," Biometrical Journal, vol. 59(1), pp. 126-144, 2017.

[209]A.Svitin, S.Malov, N.Cherkasov, P.Geerts, M.Rotkevich, P.Dobrynin, A. Shevchenko, Li Guan, J. Troyer, S. Hendrickson-Lambert, H.H. Dilks, T.K. Oleksyk, S. Donfield, E. Gomperts, D.A. Jabs, Efe Sezgin, M. Van Natta, P.R. Harrigan, Z.L. Brumme \& S.J. OBrien, "GWATCH: a web platform for automated gene association discovery analysis," GigaScience, 3:18, 2014.

[210]W. Xie, D. Agniel, A. Shevchenko, S.V. Malov, A. Svitin, N. Cherkasov, M.K. Baum, A. Campa, S. Gaseitsiwe, H. Bussmann, J.Makhema, R. Marlink, V. Novitsky, T.-H. Lee, T. Cai, S.J. O’Brien, and M. Essex, Genome-Wide Analyses Reveal Gene Influence on HIV Disease Progression and HIV-1C Acquisition in Southern Africa. AIDS Research and Human Retroviruses, vol. 33(6), pp. 597$609,2017$. 